ORIGINAL ARTICLE

\title{
A randomised controlled trial of standing programme on bone mineral density in non-ambulant children with cerebral palsy
}

\author{
J M Caulton, K A Ward, C W Alsop, G Dunn, J E Adams, M Z Mughal
}

Arch Dis Child 2004;89:131-135. doi: 10.1136/adc.2002.009316

See end of article for authors' affiliations

Correspondence to: Dr M Z Mughal, Department of Paediatric Medicine, Saint Mary's Hospital for Women and Children, Hathersage Road, Manchester M13 OJH, UK; zulf.mughal@ cmmc.nhs.uk

Accepted 12 June 2003
Background: Severely disabled children with cerebral palsy (CP) are prone to low trauma fractures, which are associated with reduced bone mineral density.

Aims: To determine whether participation in $50 \%$ longer periods of standing (in either upright or semiprone standing frames) would lead to an increase in the vertebral and proximal tibial volumetric trabecular bone mineral density (vTBMD) of non-ambulant children with CP.

Methods: A heterogeneous group of 26 pre-pubertal children with CP (14 boys, 12 girls; age 4.3-10.8 years) participated in this randomised controlled trial. Subjects were matched into pairs using baseline vertebral VTBMD standard deviation scores. Children within the pairs were randomly allocated to either intervention (50\% increase in the regular standing duration) or control (no increase in the regular standing duration) groups. Pre- and post-trial vertebral and proximal tibial vTBMD was measured by quantitative computed tomography (QCT).

Results: The median standing duration was $80.5 \%(9.5-102 \%)$ and $140.6 \%(108.7-152.2 \%)$ of the baseline standing duration in the control group and intervention group respectively. The mean vertebral VTBMD in the intervention group showed an increase of $8.16 \mathrm{mg} / \mathrm{cm}^{3}$ representing a $6 \%$ mean increase in vertebral vTBMD. No change was observed in the mean proximal tibial vTBMD.

Conclusion: A longer period of standing in non-ambulant children with CP improves vertebral but not proximal tibial vTBMD. Such an intervention might reduce the risk of vertebral fractures but is unlikely to reduce the risk of lower limb fractures in children with CP.
$\mathrm{C}$ erebral palsy (CP) is a non-progressive disorder of posture and movement due to an insult to the developing brain. Severely disabled children and adolescents with CP are prone to low trauma fracture, which occur as a result of a trivial injury or during normal activities, for example, dressing and undressing. ${ }^{1-3}$

The strength of a bone is determined by its size, the amount of mineral mass and density within the periosteal envelope, and its architecture. ${ }^{4}$ Bones constantly adapt themselves by altering the above parameters in response to mechanical forces applied to them, the largest of which comes from the action of muscles. ${ }^{4}$ In adults, bone mineral density (BMD) predicts the risk of osteoporotic fractures in the same way as blood pressure predicts the risk of stroke. ${ }^{5}$ For example, in postmenopausal women the risk for hip fracture increases by a factor of 2.6 for each age adjusted standard deviation (SD) decline in the dual energy $x$ ray absorptiometry (DXA) measured BMD of the femoral neck. ${ }^{6}$ More recently, a follow up of healthy girls with a previous forearm fracture showed that for every lSD decrease in total body BMD measured by DXA, the risk of new fracture occurring at any site doubled within four years of the initial fracture. ${ }^{7}$ Thus epidemiological data in adults and cohort follow up studies in children support the concept that low BMD is the major factor contributing to an individual's skeletal fragility.

Several studies have shown that non-ambulant children and adults with CP have reduced BMD compared to their ambulant peers. ${ }^{8-11}$ In a previous cross sectional study ${ }^{12}$ we reported that the degree of reduction in calcaneal broadband ultrasound attenuation (related to BMD and bone structure) and vertebral volumetric trabecular BMD were associated with the degree of immobility and non-weight bearing of the subjects. Nutritional factors that contribute to low BMD in these children with CP include low intake of calcium due to feeding and swallowing difficulties ${ }^{13} 14$ and increased catabolism of vitamin D in those treated with anticonvulsants. ${ }^{15}{ }^{16}$ In addition to causing pain and suffering, fractures further limit the mobility of these children leading to muscle wasting through disuse, hospitalisation, missed school, and further loss of independence.

Standing programmes are routinely used as part of a postural management approach in children with CP. A standing programme refers to the use of adaptive equipment, for example, a standing frame that provides external, adjustable support to facilitate an upright position. Standing in a developmentally appropriate position stimulates the anti-gravity muscles, increasing their strength and endurance, which also facilitates independent standing in some subjects with CP. ${ }^{17-19}$ It has been suggested that weight bearing standing programmes may be useful in maintaining $\mathrm{BMD}$ in people with $\mathrm{CP}^{20}$ Osteogenic signals from the muscles, combined with loading of the bone due to gravity, are the two possible mechanisms by which standing may stimulate bone formation and prevent bone resorption. However, there is little evidence for the use of standing programmes as a means of maintaining or improving BMD in this population. Furthermore, no guidelines exist for

Abbreviations: $\mathrm{CP}$, cerebral palsy; $\mathrm{BMD}$, bone mineral density; $\mathrm{DXA}$, dual energy $x$ ray absorptiometry; $Q C T$, quantitative computed tomography; RCT, randomised controlled trial; RNI, reference nutrient intake; SDS, standard deviation score; VTBMD, volumetric trabecular bone mineral density 


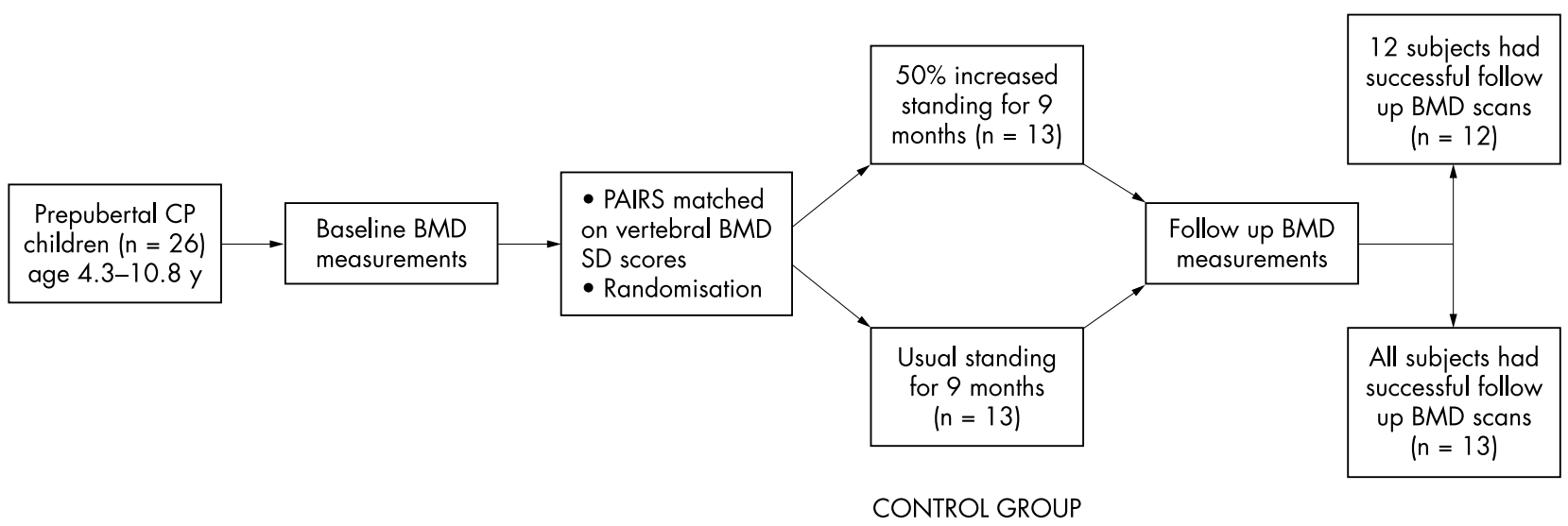

Figure 1 The study design.

standing children with CP so individual health professionals (usually physiotherapists) are left to decide the frequency, duration, type of standing regimen, and frame, based on their clinical experience.

The aim of this randomised controlled trial (RCT) was to determine whether participation in 50\% longer periods of standing (in either upright or semi-prone standing frames) during one school academic year would lead to an increase in vertebral and proximal tibial volumetric trabecular bone mineral density (vTBMD) of non-ambulant children with CP.

\section{PARTICIPANTS AND METHODS Subjects}

The study subjects were recruited from from schools for children with special educational needs in the Greater Manchester area. Non-ambulant CP children were included in the study if they were pre-pubertal, already involved in a standing programme, and able to tolerate an increased standing duration. Exclusion criteria included fracture and immobilising bone or soft tissue release surgery of the lower limbs within 12 months of the start of the trial. Pubertal status of each subject was determined by the grading system of Tanner. ${ }^{21}$ The trial was approved by the relevant local ethical committees and the North West Multi-Centre Research Ethics Committee. Informed written consent was obtained from the person with parental responsibility for the child.

\section{Sample size and recruitment}

The sample size was based on the mean vertebral vTBMD and calcaneal broadband ultrasound attenuation standard deviation scores obtained from a previous cross sectional study. ${ }^{12}$ The original grant was awarded on the basis of recruiting a total of 50 non-ambulant CP children (25 in the intervention group and 25 receiving the routine standing programme) in order to show a $10 \%$ difference in vertebral vTBMD with an $\alpha$ of 0.05 and $80 \%$ power. This effect size was arbitrarily chosen as the clinically important difference. After the start of the RCT, it became clear that the recruitment of the required number of subjects was not going to be possible from special education needs schools in the Greater Manchester area. Due to these recruitment difficulties the funding body and ethical committees granted our request to modify the original proposal to a pilot RCT.

Fifty nine potential subjects for the trial were identified by consultant community paediatricians and paediatric physiotherapists from three community health care Trusts in the Greater Manchester area. However, 21 of the parents approached declined to take part in the study, and a further 12 did not satisfy the inclusion criteria. Therefore 26 children, 14 boys and 12 girls, aged 7.32 (1.8) years (range 4.33-10.83) participated in the trial. Four children in the intervention group and two in the control group were receiving anticonvulsants during the trial; one child in each group was on baclofen. The children whose parents refused to participate in the RCT had similar characteristics to the participants. We therefore believe that the participants were representative of the general non-ambulant childhood CP population. The authors defined the standing programme as a monitored period of standing in a standing frame while participating in usual classroom activities. Such programmes are administered by a variety of upright or semi-prone standing frames with each child being assisted and secured into the standing frame. The optimum period of standing for each child was determined by their physiotherapist and during the trial, specifically appointed carers assisted physiotherapists to monitor the duration of standing period.

\section{Randomisation and the trial protocol}

Figure 1 shows the trial protocol. The RCT took place during one school academic year (nine months), between September 1999 and July 2000. The pre-trial duration of standing was determined for each subject over a six week period prior to the start of the trial and expressed as the mean standing period in minutes per week. Subjects were matched into pairs using baseline vertebral volumetric trabecular bone mineral density (vTBMD) standard deviation scores (SDS), calculated using the only available reference data collected in healthy 2-19 year old North American Caucasian subjects. ${ }^{22}$ The trial statistician randomly allocated each child within the pairs to either the intervention $(50 \%$ increase in the regular standing duration) or the control (no increase in the regular standing duration) group. The standing programme during the trial was specific for each subject. For those randomised to the intervention limb of the trial, the increased standing period was fitted around curriculum activities within each classroom; it was achieved by either increasing the duration during each standing session or the frequency of standing sessions. Throughout the trial, each child's daily standing duration was measured using digital timers and recorded in standing diaries. The mean standing period during the trial for each subject was expressed as percentage of their baseline or the pre-trial standing period (mean number of minutes of standing per week during the trial $\div$ mean number of minutes of standing per week prior to the trial $\times 100)$. 


\section{Nutritional analysis}

Food intake was recorded using standardised three day (two weekdays and one weekend day) food diaries $(n=21)$ or food frequency questionnaires administered to parents by telephone $(n=5)$. The daily intake of calcium $(\mathrm{mg})$ was estimated twice during the trial (pre-trial and at eight months) using the Comp-Eat v.5 for Windows Nutritional Software (CompEat Nutrition Systems, Closterworth, Grantham, UK). The mean calcium intake (mg/day) during the trial was expressed as a percentage of the United Kingdom recommended reference nutrient intakes (RNI) for the age and gender of the subject. ${ }^{23}$

\section{Length and weight estimations}

Length $(\mathrm{cm})$ was estimated in the supine lying position using a steel tape measure. Accurate length measurements were not possible to measure due to contractures and mild scoliotic deformities of the spine. Weight $(\mathrm{kg})$ was measured with outer clothes and shoes removed using the sit-down scales available at each school.

\section{Outcome measures}

The outcome measures of this trial were vertebral and proximal tibial vTBMD, expressed in $\mathrm{mg} / \mathrm{cm}^{3}$, which were measured using the Philips Medical Systems 4000 SR Tomoscan spiral quantitative computed tomography (QCT) scanner (Philips Medical Imaging, Best, Netherlands), in conjunction with three dimensional (3D) QCT-Pro software (Mindways, San Francisco, USA). QCT is able to selectively assess the VTBMD at axial and appendicular skeletal sites without influence from body size, which is a major advantage of this technique when used to measure BMD in children. ${ }^{24}$ The positioning and scanning was carried out with parents/ carers and staff assisting each child to lie still without the need for sedation. The total duration of scanning for each child was < 10 minutes. A single energy, low dose ( $120 \mathrm{kv}$; $50 \mathrm{~mA}$ ) scanning technique was used and calibration was achieved by simultaneous scanning of a bone mineral reference calibration phantom consisting of varying concentrations of dipotassium hydrogen phosphate solution. The sites to be scanned were located by digital "scout" projection radiographs. A "block" of data consisting of up to $30,3 \mathrm{~mm}$ contiguous slices, with approximately two seconds per slice, were obtained at each site. Vertebral TBMD (vTBMD) measurements were made at central portion of vertebral bodies, devoid of their cortical envelopes and neural arches. ${ }^{25}$ The proximal tibial VTBMD was measured at a site rich in trabecular bone, distal to the tibia-fibular junction just below the tibial plateau away from the growth plate, using an inhouse protocol. Due to the relatively small size of the trabeculae compared to the size of a CT pixel, the apparent VTBMD at these sites reflects not only the amount of mineralised bone within trabeculae but also the amount of bone marrow per pixel. ${ }^{26} 27$

The location of the region of interest, consisting of a cube of trabecular bone within digitally stored "blocks" of data from vertebral and proximal tibial sites, was undertaken manually. The root mean square precision $(\mathrm{CV} \%)^{28}$ of this operator dependent process was determined at these sites. The CV\% for tibial vTBMD measurement was $2.1 \%$, whereas that of vertebral vTBMD was $0.9 \%$. The total effective radiation dose for the scans was $85 \mu \mathrm{Sv} ; 55 \mu \mathrm{Sv}$ for vertebrae and $30 \mu \mathrm{Sv}$ for the tibia.

\section{Blinding}

Due to the overt nature of the intervention, only the investigators responsible for measuring and analysing vTBMD were "blinded" to which children were in the intervention and control groups.

\section{Statistical analyses}

Statistical analyses were carried out using Stata version 6.0 (StataCorp, 1999). The changes in measured vertebral and proximal tibial vTBMD $\left(\mathrm{mg} / \mathrm{cm}^{3}\right)$ were assessed through a random effects model using the Stata command xtreg. The vertebral vTBMD data from the L2 vertebral body was used in the analysis, as good quality pre- and post-trial scans were available for this vertebra. Random variation was allowed for at the level of the individual child and matched pair (that is, allowing for the matched design). As specified in the original grant protocol, the model included the following individual level covariates (treated as a qualitative factor where appropriate): type of cerebral palsy, baseline standing duration, type of standing, and the baseline average daily calcium intake. The results were analysed on the basis of intention to treat, in which vertebral and proximal tibial vTBMD of all the subjects were analysed with the group to which they were randomised, whether or not they completed the trial protocol.

\section{RESULTS}

Table 1 shows characteristics of the subjects. The mean age of subjects in the control group (7.31 (SD 2) years) was not different from that of subjects in the intervention group (7.32 (SD 1.8) years). In the control group the median standing duration (range) was $80.5 \%(9.5-102 \%)$ of the baseline standing duration. In the intervention group the median standing duration (range) was $140.6 \%$ (108.7-152.2\%) of the baseline standing duration. Thus the net difference in standing period between the two groups was approximately $60 \%$. Estimated daily calcium intake ( $\mathrm{mg} /$ day) during the trial (\% RNI) in the control and intervention groups were 790 (SD 273) [154\% (SD 54\%)] and 717 (SD 218) [138\% (SD $35 \%)]$, respectively.

In one child in the intervention group, we were unable to obtain satisfactory follow up (post-trial) vTBMD measurements due to movement artefacts. The vertebral vTBMD in the intervention group showed an increase of $8.91 \mathrm{mg} / \mathrm{cm}^{3}$ compared to the control group (95\% CI 2.40 to 15.41 ; $p=0.007)$. The intention to treat analysis, including the subject in whom the post-trial vertebral vTBMD scan was unsuccessful (with his pre-trial vertebral vTBMD value carried forward), yielded a group difference of $8.16 \mathrm{mg} / \mathrm{cm}^{3}$ (95\% CI 1.93 to $14.39 ; \mathrm{p}=0.01$ ). This represents a $6 \%$ mean increase in vertebral vTBMD in the intervention group. The proximal tibial VTBMD in the intervention group showed a change of $-0.85 \mathrm{mg} / \mathrm{cm}^{3}$ compared to the control group (95\% CI -16.83 to $15.13 ; \mathrm{p}=0.92$ ) when analysed using the same strategy as for the vertebral site.

\section{DISCUSSION}

To the best of our knowledge this is the first RCT investigating the benefits of a longer period of standing on BMD in non-ambulant children with CP. We used 3D QCT to selectively measure VTBMD at the proximal tibial and vertebral sites. An important advantage of QCT measured vTBMD is that it is independent of skeletal size, ${ }^{24}$ which is important when assessing the BMD of children, whose bone size may vary with age, height, and weight. The main finding of this trial was that after allowing for the effects of prestated confounding variables, a longer period of standing resulted in a significant increase in the vertebral but not proximal tibial vTBMD. Chad et al observed a 5.6\% increase in femoral neck volumetric BMD in nine children with spastic $\mathrm{CP}$ who were treated with load bearing physical activities for eight months, compared with a $-6.3 \%$ change in the control group. ${ }^{29}$ However, increased physical activity was not a treatment option for non-ambulant CP children that we studied. The results of the current RCT are diametrically 
Table 1 Subject characteristics

\begin{tabular}{|c|c|c|c|c|c|c|c|}
\hline Age (y) & Gender* & $\begin{array}{l}\text { Type of } \\
\text { standingt }\end{array}$ & $\begin{array}{l}\text { Type of cerebral } \\
\text { palsy } \ddagger\end{array}$ & $\begin{array}{l}\text { Randomisation } \\
\text { group§ }\end{array}$ & $\begin{array}{l}\text { Mean no. of minutes } \\
\text { of standing per week } \\
\text { prior to the trial }\end{array}$ & $\begin{array}{l}\text { Mean no. of minutes } \\
\text { of standing per week } \\
\text { during the trial }\end{array}$ & $\begin{array}{l}\text { Duration of standing during } \\
\text { the trial as } \% \text { of the pre-trial } \\
\text { standing duration }\end{array}$ \\
\hline 7.5 & $M$ & 1 & $3 Q$ & C & 240 & 182 & $95 \%$ \\
\hline 6.0 & $\mathrm{~F}$ & 1 & $1 Q$ & C & 240 & 172 & $90 \%$ \\
\hline 8.5 & $M$ & 1 & $1 Q$ & 1 & 194 & 273 & $141 \%$ \\
\hline 9.4 & $\mathrm{~F}$ & 1 & $2 Q$ & C & 225 & 42 & $46 \%$ \\
\hline 5.8 & $\mathrm{~F}$ & 2 & $1 Q$ & C & 260 & 156 & $75 \%$ \\
\hline 5.1 & $M$ & 2 & $4 Q$ & C & 240 & 174 & $91 \%$ \\
\hline 7.8 & M & 2 & $4 Q$ & 1 & 180 & 219 & $152 \%$ \\
\hline 4.3 & $\mathrm{~F}$ & 2 & $1 Q$ & i & 240 & 362 & $151 \%$ \\
\hline 8.5 & $\mathrm{~F}$ & 2 & $2 Q$ & 1 & 234 & 351 & $150 \%$ \\
\hline 7.3 & $M$ & 2 & $2 Q$ & C & 325 & 307 & $95 \%$ \\
\hline 5.3 & $M$ & 1 & $1 \mathrm{D}$ & I & 336 & 406 & $121 \%$ \\
\hline 7.0 & M & 1 & $1 Q$ & C & 228 & 166 & $91 \%$ \\
\hline 10.8 & $M$ & 2 & $1 Q$ & C & 400 & 15 & $9.5 \%$ \\
\hline 7.0 & M & 2 & $4 \mathrm{D}$ & I & 360 & 560 & $130 \%$ \\
\hline 4.3 & $M$ & 2 & $1 Q$ & C & 240 & 240 & $83 \%$ \\
\hline 8.7 & $\mathrm{~F}$ & 2 & $1 Q$ & $\mathrm{C}$ & 195 & 120 & $102 \%$ \\
\hline 9.1 & $M$ & 2 & $1 Q$ & I & 300 & 453 & $151 \%$ \\
\hline 7.8 & $\mathrm{~F}$ & 2 & $1 Q$ & 1 & 210 & 320 & $152 \%$ \\
\hline 10.2 & $M$ & 2 & $1 Q$ & C & 400 & 270 & $84 \%$ \\
\hline 10.7 & $M$ & 2 & $1 Q$ & I & 216 & 272 & $126 \%$ \\
\hline 5.3 & $\mathrm{~F}$ & 2 & $3 Q$ & i & 240 & 365 & $152 \%$ \\
\hline 7.2 & $M$ & 1 & $1 Q$ & C & 240 & 189 & $98 \%$ \\
\hline 6.0 & $\mathrm{~F}$ & 2 & $1 \mathrm{D}$ & C & 280 & 196 & $87 \%$ \\
\hline 8.3 & $\mathrm{~F}$ & 2 & $1 Q$ & 1 & 180 & 265 & $147 \%$ \\
\hline 6.1 & $\mathrm{~F}$ & 2 & $1 Q$ & I & 300 & 326 & $109 \%$ \\
\hline 6.6 & $\mathrm{~F}$ & 1 & $2 Q$ & i & 384 & 675 & $146 \%$ \\
\hline
\end{tabular}

${ }^{*} M$, male; $F$, female

$\dagger 1$, semi-prone standing; 2 , upright standing.

$\ddagger 1$, spasticity; 2 , athetoid with spasticity; 3 , athetoid or ataxic; 4 , predominately low or variable tone but no athetosis or ataxia. $Q$, quadruplegia; $D$, diplegia. §l, intervention; C, control.

opposite to those reported by Ward and colleagues, ${ }^{30}$ who randomised a heterogeneous group of children with disabling conditions to standing on either active or placebo vibrating platforms, for 10 minutes per day, five times a week for six months. The active vibrating platforms generated low magnitude $(0.3 \mathrm{G})$, high frequency $(90 \mathrm{~Hz})$ mechanical stimuli, whereas placebo platforms produce a $500 \mathrm{~Hz}$ audible tone, similar to that produced by active platforms. A highly significant net increase in tibial $\left(15.72 \mathrm{mg} / \mathrm{cm}^{3} ; 95 \%\right.$ CI 6.57 to $24.87 ; \mathrm{p}=0.003$ ) but not vertebral vTBMD was observed in children who stood on the active vibrating platforms.

The improvement in vertebral VTBMD shown by this trial need to be appreciated with respect to alternative treatments for improving BMD in children with CP. Bisphosphonates, which inhibit osteoclastic bone resorption, have been shown to increase vTBMD by $20-40 \%$ after $12-18$ months of treatment in three non-ambulant children with $\mathrm{CP} .{ }^{10}$ In a small placebo controlled RCT, Henderson et al observed that the distal femoral metaphyseal BMD increased by $89 \%$ (SD $21 \%$ ) in six children with quadriplegic CP treated with intravenous pamidronate for 18 months, compared with a $9 \%$ (SD 6\%) increase in the placebo group. ${ }^{31}$

The important question to consider is whether the observed increase in vertebral vTBMD would translate into increased bone strength, and more importantly, decreased fracture risk. This question can only be answered by a long term cohort follow up study of increased duration of standing in CP children, with the cumulative incidence of fractures included as one of the trial outcome measures. However, we can speculate about the potential beneficial effects of the observed increase in vertebral vTBMD by comparing our results with those of studies in adults and in laboratory animals that have looked at the association between increase in vertebral BMD and bone strength, or reduction in the incidence of osteoporotic fractures. In ovariectomised baboons treated with the aminobisphosphonate alendronate, the vertebral compressive strength of vertebrae increased with the square of BMD. ${ }^{32}$ Thus a small increase in vertebral $\mathrm{BMD}$ results in a much greater increase in vertebral biomechanical strength. Treatment of postmenopausal women with risedronate, an oral bisphosphonate, for three years resulted in a $5.4 \%$ increase in vertebral bone density and a $41 \%$ reduction in the incidence of new vertebral fractures. ${ }^{33}$ Thus, it is plausible that the increase in vertebral vTBMD due to longer periods of standing in non-ambulant children with CP would result in increased bone strength and lower risk of vertebral fractures. Vertebral fractures in these children tend to occur insidiously, often presenting as kyphosis.

Lack of improvement of proximal tibial VTBMD in response to longer periods of standing suggests that this intervention is not likely to reduce the incidence of metaphyseal and submetaphyseal fractures in non-ambulant children with CP. The most common site of low trauma fracture seen in these children is in the diaphysis of long bones. The bending strength of a long bone diaphysis increases as the cortical bone mass is distributed further away from the centre of the bone shaft. ${ }^{34}$ Therefore, improvement in the strength of the diaphysis of long bones would require an intervention or treatment that caused an increase in the bone diameter through periosteal and endocortical expansion. These outcomes were not studied as part of this RCT, which was specifically designed to measure VTBMD at the proximal tibial and vertebral sites.

In summary, the results of this pilot RCT suggest that a longer period of standing leads to a significant increase in vertebral but not proximal tibial vTBMD in non-ambulant CP children. As BMD is a surrogate for bone strength, ${ }^{57}$ we believe that this intervention might reduce the risk of vertebral fractures in mobility impaired children with CP. However, from the results of this trial we conclude that longer duration of standing is not likely to reduce the risk of lower limb long bone fractures in children with CP. Therefore, on present evidence, a longer period of standing 
cannot be recommended for the prevention of long bone fractures in non-ambulant children with CP. Further RCTs of physical or pharmacological treatments with fracture prevention as the primary end point are required in this group of subjects.

\section{ACKNOWLEDGEMENTS}

This work was supported by a Grant (PCD2/Al/306) from the NHS R\&D Programme for People with Physical and Complex Disabilities. We are grateful to Dr Patrick Doherty, Dr Wendy Rankin, Dr Ian McKinley, Dr Robert Downes, Alison Balkas, Patsy Potts, Jean Menzies, Janet Ward, Sally-Anne Attwood, Melanie Littler, Sue Walmsley, Lyn Moody, Sue Rawlinson, and Gisela Taylor for their invaluable help with this trial.

MZM designed and supervised the trial. JMC was responsible for day to day coordination of the trial. CWA, KAW, and JEA were responsible for measuring and analysing bone mineral density. GD was responsible for randomisation and data analysis. MZM and JMC wrote the first draft of the manuscript and other investigators contributed to preparation of the final draft. MZM will act as guarantor.

\section{Authors' affiliations}

J M Caulton, The Manchester School of Physiotherapy, Manchester Royal Infirmary, Manchester, UK

K A Ward, C W Alsop, J E Adams, Clinical Radiology, Imaging Science \& Biomedical Engineering, University of Manchester, UK

G Dunn, School of Epidemiology \& Health Sciences, University of Manchester, UK

M Z Mughal, Department of Paediatric Medicine, Saint Mary's Hospital for Women and Children, Manchester, UK

\section{REFERENCES}

1 Lee JJ, Lyne E, eds. Pathologic fractures in severely handicapped children and young adults. J Pediatr Orthop 1990;10:497-500.

2 Lingham S, Joester J. Spontaneous fractures in children and adolescents with cerebral palsy. BMJ 1994;309:265

3 Brunner R, Doderlein L. Pathological fractures in patients with cerebral palsy. J Pediatr Orthop B 1996;5:232-8.

4 Frost HM. On our age-related bone loss: insights from a new paradigm. J Bone Miner Res 1997;12:1539-46.

5 Marshall D, Johnell O, Wedel H. Meta-analysis of how well measures of bone mineral density predict occurrence of osteoporotic fractures. BMJ 1996;312:1254-9.

6 De Laet CE, Van Hout BA, Burger H, et al. Hip fracture prediction in elderly men and women: validation in the Rotterdam study. J Bone Miner Res 1998:13:1587-93.

7 Goulding A, Jones IE, Taylor RW, et al. 2000 More broken bones: a 4-year double cohort study of young girls with and without distal forearm fractures. J Bone Miner Res 2000;15:2011-18.

8 Henderson RC, Lin PP, Greene WB. Bone-mineral density in children and adolescents who have spastic cerebral palsy. J Bone Joint Surg Am 1995;77:1671-81.

9 Henderson RC. Bone density and other possible predictors of fracture risk in children and adolescents with spastic quadriplegia. Dev Med Child Neurol 1997:39:224-7.
10 Shaw NJ, White CP, Fraser WD, et al. Osteopenia in cerebral palsy. Arch Dis Child 1994;71:235-8.

11 Tasdemir HA, Buyukavci M, Akcay F, et al. Bone mineral density in children with cerebral palsy. Pediatr Int 2001;43:157-60.

12 Wilmshurst S, Ward K, Adams JE, et al. Mobility status and bone density in cerebral palsy. Arch Dis Child 1996;75:164-5.

13 Duncan B, Barton LL, Lloyd J, et al. Dietary considerations in osteopenia in tube-fed nonambulatory children with cerebral palsy. Clin Pediatr (Phila) 1999:38:133-7.

14 Shetty AK, Carlin J, Mughal MZ. Urinary calcium excretion in enterally fed disabled children. Arch Dis Child 2001;85:58-9.

15 Nilsson OS, Lindholm TS, Elmstedt E, et al. Fracture incidence and bone disease in epileptics receiving long-term anticonvulsant drug treatment. Arch Orthop Trauma Surg 1986:105:146-9.

16 Tolman KG, Jubiz W, Sannella JJ, et al. Osteomalacia associated with anticonvulsant drug therapy in mentally retarded children. Pediatrics 1975;56:45-50.

17 Green EM, Mulcahy CM, Pountney TE, et al. The Chailey standing support for children and young adults with motor impairment: a developmental approach. British Journal of Occupational Therapy 1993;56:13-18.

18 Nelson DL, Schau EM. Effects of a standing table on work productivity and posture in an adult with developmental disabilities. Work 1997;9:13-20.

19 Gordon A. The Chailey standing support. British Journal of Occupational Therapy 1993;56:150-1.

20 Stuberg WA. Considerations related to weight-bearing programs in children with developmental disabilities. Phys Ther 1992;72:35-40.

21 Tanner JM. Growth at adolescence, 2nd edn. Oxford: Blackwell Scientific, 1962:28-39.

22 Gilsanz V, Gibbens DT, Roe TF, et al. Vertebral bone density in children: effect of puberty. Radiology 1988;166:847-50.

23 Department of Health. Nutrition and one health: with particular reference to calcium and vitamin D, Report on health and social subjects; 49. London: HMSO, 1998.

24 Gilsanz V. Bone density in children: a review of the available techniques and indications. Eur J Radiol 1998;26, 2:177-82.

25 Cann CE, Genant HK. Precise measurement of vertebral mineral content using computed tomography. Journal of Computer Assisted Tomography 1980:4:493-500

26 Genant HK, Engelke K, Fuerst T, et al. Noninvasive assessment of bone mineral and structure: state of the art. J Bone Miner Res 1996;11:707-30.

27 Gilsanz V, Roe TF, Mora S, et al. Changes in vertebral bone density in black girls and white girls during childhood and puberty. N Engl J Med 1991;325:1597-600

28 Gluer CC, Blake G, Lu Y, et al. Accurate assessment of precision errors: how to measure the reproducibility of bone densitometry techniques. Osteoporos Int 1995;5:262-70.

29 Chad KE, Bailey DA, McKay HA, et al. The effect of a weight-bearing physical activity program on bone mineral content and estimated volumetric density in children with spastic cerebral palsy. J Pediatr 1999;135:115-17.

30 Ward K, Alsop C, Brown S, et al. A randomized, placebo controlled, pilot trial of low magnitude, high frequency loading treatment on low bone mineral density in children with disabling conditions. J Bone Miner Res. In press.

31 Henderson RC, Lark RK, Kecskemethy HH, et al. Bisphosphonates to treat osteopenia in children with quadriplegic cerebral palsy: a randomized, placebo-controlled clinical trial. J Pediatr 2002;141:644-51.

32 Balena $\mathbf{R}$, Toolan BC, Shea $M$, et al. The effects of 2 -year treatment with the aminobisphosphonate alendronate on bone metabolism, bone histomorphometry, and bone strength in ovariectomized nonhuman primates. J Clin Invest 1993;92:2577-86.

33 Harris ST, Watts NB, Genant HK, et al. Effects of risedronate treatment on vertebral and nonvertebral fractures in women with postmenopausal osteoporosis: a randomized controlled trial. Vertebral Efficacy With Risedronate Therapy (VERT) Study Group. JAMA 1999;282:1344-52.

34 Seeman E. Sexual dimorphism in skeletal size, density, and strength. J Clin Endocrinol Metab 2001;86:4576-84. 